\section{Serum albumin concentrations and oedema in the newborn}

Sir,

Cartlidge and Rutter have provided useful data relating serum albumin concentration to gestation and discuss its poor correlation with clinical oedema. ${ }^{1}$ Their view, however, that 'hypoalbuminaemia of prematurity' is a 'normal not a pathological finding' is debatable. A biochemical or clinical finding that is consistently associated with prematurity is not necessarily 'normal' and may indeed be undesirable. In this study the authors have examined only one clinical aspect of hypoalbuminaemia, oedema; yet low serum albumin concentration may have diverse effectsfor instance, on drug and bilirubin binding and toxicity.

Currently, we are analysing the relation between clinical outcome and plasma albumin concentration measured in over 4000 samples during a longitudinal study of 932 infants. Preliminary multiple regression analysis has reaffirmed the strong association between hypoalbuminaemia and respiratory disease, even after adjustment for gestation. Low plasma albumin concentration in this circumstance cannot be regarded as 'normal' and probably reflects excessive losses-for example, into alveoli-and the adverse effect of severe disease or suboptimal nutrition on protein synthesis by an immature liver. Yet sick infants must have comprised an appreciable proportion of the low gestation sample investigated by Cartlidge and Rutter.

These workers contend that measurement of serum albumin concentrations in preterm infants, either routinely or in the presence of oedema, is of little use in their management'. This conclusion might be misinterpreted. A low plasma albumin concentration may be used as a marker for the high risk neonate, may influence the interpretation of other biochemical findings, and may warrant intervention in its own right. Albumin estimation is cheap and, I believe, should not be abandoned as a routine investigation in very low birthweight infants in the neonatal period.

\section{Reference \\ 1 Cartlidge P, Rutter N. Serum albumin concentrations and oedema in the newborn. Arch Dis Child 1986:61:661-5.}

\section{A Lucas \\ University of Cambridge and MRC Dunn Nutrition Unit, Cambridge CB4 $1 X J$}

Drs Rutter and Cartlidge comment:

We agree with $\mathrm{Dr}$ Lucas that a finding consistently associated with prematurity is not necessarily normal. We found though that serum albumin was a poor marker of illness in low gestation infants: concentrations were low in both sick and well infants. His unpublished study clearly contains many more infants and we look forward to seeing it. We still contend that routine measurement of serum albumin is of little use in the management of the preterm infant. Certainly, bilirubin and drug binding are affected by low serum albumin concentrations, but routine measurements are not carried out with this in mind. Perhaps a case can be made for routinely measuring albumin whenever serum calcium concentrations are measured in the preterm infant. Hypocalcaemia is commonly diagnosed and treated on the basis of low uncorrected calcium concentrations, but it is largely an artefact caused by a low serum albumin concentration.

\section{Minimal change nephrotic syndrome and cyclophosphamide}

Sir,

The interesting annotation by Trompeter about minimal change nephrotic syndrome and cyclophosphamide rightly indicates that the use of alkylating agents is controversial in a 'non-malignant condition with an excellent long term prognosis'.'

The point is well made that an increased risk of malignant disease after immunosuppressive drugs has been observed in both adults and children, but this is perhaps too broad a generalisation. It is possible to go further. Cyclophosphamide, chlorambucil, and nitrogen mustard all undoubtedly cause secondary leukaemias. ${ }^{2} 3$ Other immunosuppressive agents, such as methotrexate or azathioprine, apparently do not. In other words not all cytostatic immunosuppressants carry the same risk of inducing neoplasia. While I appreciate that they may not have an equivalent therapeutic effect either, nevertheless it is an important point that should be borne in mind by clinicians who may be tempted to prescribe one for any benign disorder.

J S Lilleyman

The Children's Hospital Sheffield

\section{References}

1 Trompeter RS. Minimal change nephrotic syndrome and cyclophosphamide. Arch Dis Child 1986;61:727-9.

2 International Agency for Research on Cancer. Monographs on the carcinogenic risk of chemicals to humans. Suppl 4. Lyon: IARC, 1982.

${ }^{3}$ Rosner F, Grunwald H. Cytotoxic drugs and leukaemogenesis. Clin Haematol 1980;9:663-81.

\section{High dose intravenous methylprednisolone in treatment of recessive osteopetrosis}

Sir,

The paper of Dorantes et al ${ }^{1}$ gives me an opportunity to report my results in treating recessive osteopetrosis with high dose intravenous methylprednisolone, which partially support their results.

Three infants, a 4 month old girl, a 5 month old boy, and, more recently, a 14 month old girl, with typical recessive osteopetrosis were treated with intravenous 